\title{
WEIGHTED COMPOSITIONS
}

\section{Moser and E. L. Whitney}

(received October 17, 1960)

By a composition of a positive integer $\mathrm{n}$ is meant a representation of $n$ as a sum of one or more positive integers where the order of the summands is taken into account. Thus for example 4 has the eight compositions $4=3+1=1+3$ $=2+2=2+1+1=1+2+1=1+1+2=1+1+1+1$. Now $\mathrm{n}$ can be written in the form $1+1+\ldots+1$ with $\mathrm{n}-1$ plus signs. Deletion of any subset of the se plus signs breaks $n$ into parts which form a composition of $n$. Conversely, any composition of $n$ corresponds to a subset of plus signs, so that the number of compositions of $n$ is the number of subsets of a set with $\mathrm{n}-1$ elements, namely $2^{\mathrm{n}-1}$. In this note we obtain a number of generalizations of this rather obvious remark by making use of the notion of a weighted composition and the method of generating series.

Let $\{w\}: w_{1}, w_{2}, w_{3}, \ldots$ be a sequence of numbers called weights. Corresponding to such a sequence we define another sequence $\{w\}: w_{1}, w_{2}, w_{3}, \ldots$ by

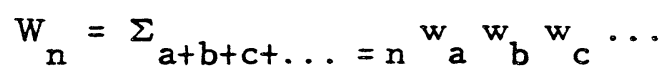

where the sum is taken over all compositions of $n$. If $w_{n}=1$ for $n=1,2,3, \ldots$ then $W_{n}$ will simply be the number of compositions of $n$ which is, as we have seen, $2^{n-1}$. We will consider,more generally, relations between $\{w\}$ and $\{W\}$.

We form the power series generating functions for $\{w\}$ and $\{W\}$ namely

$$
\begin{aligned}
& w(x)=\Sigma_{n=1}^{\infty} w_{n} x^{n} \\
& w(x)=\Sigma_{n=1}^{\infty} w_{n} x^{n}
\end{aligned}
$$

Canad. Math. Bull. vol. 4, no. 1, Janua ry 1961 
and prove

THEOREM 1.

and

$$
W(x)=\frac{w(x)}{1-w(x)}
$$

$$
w(x)=\frac{W(x)}{1+W(x)} \text {. }
$$

The two parts of the theorem are clearly equivalent so we prove only the first. Note that the coefficient of $x^{n}$ in $(w(x))^{k}=\left(w_{1} x^{1}+w_{2} x^{2}+\ldots\right)^{k}$ is the sum of terms $w_{a} w_{b} w_{c} \ldots$ where $a+b+c+\ldots=n$ and the number of summands in this last sum is $k$. Thus from (1) it follows that

$$
W(x)=\sum_{k=1}^{\infty}(w(x))^{k}=\frac{w(x)}{1-w(x)}
$$

as required.

From the second form of theorem 1 it follows that

$$
w(x)=-\Sigma_{k=1}^{\infty}(-w(x))^{k}
$$

and by comparing coefficients of $x^{n}$ in (5) we obtain

\section{THEOREM 2}

$$
w(n)=\Sigma_{a+b+c+\ldots=n}-\left(-w_{a}\right)\left(-w_{b}\right)\left(-w_{c}\right) \ldots
$$

This result affords an inversion of (1) and our theorems reveal that in general the sequence $\{w\}$ can be determined from the sequence $\{W\}$ as easily as conversely.

We consider next a few simple examples of the use of theorem 1 .

EXAMPLE 1. $\mathrm{w}_{\mathrm{n}}=1, \mathrm{n}=1,2,3, \ldots$

Here $w(x)=x+x^{2}+x^{3}+\ldots=x /(1-x)$ and $W(x)=x /(1-2 x)=$ $x+2 x^{2}+2^{2} x^{3}+\ldots+2^{n-1} x^{n}+\ldots$ so that $w_{n}=2^{n-1}$ as has already been noted.

$$
\text { EXAMPLE 2. } w_{n}=(-1)^{n+1} .
$$


Here $w(x)=x-x^{2}+x^{3}-\ldots=x /(1+x)$ and $w(x)=x$. Thus $W_{1}=1$ and $w_{n}=0$ for $n>1$. Interpreting this result in the light of (1) reveals that for $n>1$ the number of compositions of $\mathrm{n}$ involving an even number of odd parts is the same as the number of compositions of $\mathrm{n}$ involving an odd number of odd parts. This result can also be obtained by a direct combinatorial a rgument.

$$
\text { EXAMPLE 3. } \quad \mathrm{w}_{\mathrm{n}}=\mathrm{n} \text {. }
$$

Now $w(x)=x+2 x^{2}+3 x^{3}+\ldots=x /(1-x)^{2}$ and

$$
W(x)=\sum_{n=1}^{\infty} W_{n} x^{n}=x /\left(1-3 x+x^{2}\right) .
$$

Cross multiplying in (6) and comparing coefficients of $x^{n}$ gives $\mathrm{w}_{1}=1, \mathrm{w}_{2}=3$ and $\mathrm{w}_{\mathrm{n}+2}=3 \mathrm{w}_{\mathrm{n}+1}-\mathrm{w}_{\mathrm{n}}$ for $\mathrm{n}>0$, which determines $\{W\}$. We leave it to the reader to identify these numbers with a subset of the Fibonacci numbers defined by $f_{0}=f_{1}=1, f_{n+2}=f_{n+1}+f_{n}(n \geq 0)$. In fact it is readily proved in this case that $W_{n}=f_{2 n-1}$ and that an explicit formula for $W_{n}$ is given by

$$
\mathrm{w}_{\mathrm{n}}=\frac{1}{\sqrt{5}}\left[\left(\frac{1+\sqrt{5}}{2}\right)^{2 \mathrm{n}}-\left(\frac{1-\sqrt{5}}{2}\right)^{2 \mathrm{n}}\right] .
$$

$$
\text { EXAMPLE 4. } \quad \mathrm{w}_{\mathrm{n}}=\mathrm{n} \text {. }
$$

Here $W(x)=x+2 x^{2}+3 x^{3}+\ldots=x /(1-x)^{2}$ and $w(x)=\frac{x}{1-x+x^{2}}$. Thus $w(x)=\frac{x+x^{2}}{1-x^{3}}=x+x^{2}-x^{4}-x^{5}+x^{7}+x^{8}-\ldots$ so that

$\{w\}$ is a periodic sequence of period 6 which begins $1,1,0,-1$, $-1,0, \ldots$. Interpreting this result in the light of (1) yields the following theorem for which we have not been able to find a direct combinatorial proof.

THEOREM 3. The number of compositions of $n$ into parts $\equiv 1,2,4$ or $5(\bmod 6)$ and involving an even number of parts $\equiv 4$ or $5(\bmod 6)$ exceeds by $n$ the number of compositions of $n$ into parts $\equiv 1,2,4$ or $5(\bmod 6)$ and involving an odd number of parts $\equiv 4$ or $5(\bmod 6)$.

The preceding examples are all special cases of 
THEOREM 4. A linear recurrence relation

$$
a_{0} w_{i}+a_{1} w_{i+1}+\ldots+a_{m} w_{i+m}=0 \quad\left(i>0, a_{m} \neq 0\right)
$$

among the weights in $\{w\}$ induces and is induced by such a relation among the elements of $\{W\}$.

Indeed if

$$
A_{m}(x)=a_{0} x^{m}+a_{1} x^{m-1}+\ldots+a_{m},
$$

then

(10) $A_{m}(x) w(x)-a_{0} w(x) x^{m}=\Sigma_{j=0}^{m-1} x^{m-j} \Sigma_{i=j}^{m-1} a_{i+1} w_{i+1-j}$, and

(11) $A_{m}(x) w(x)=\dot{B}_{m}(x)=b_{0} x^{m}+b_{1} x^{m-1}+\ldots+b_{m-1} x$ where

$$
a_{j+1} w_{1}+a_{j+2} w_{2}+\ldots+a_{m} w_{m-j}=b_{j}, \quad j<m,
$$

from the form of the Cauchy product of power series. Now

so that

$$
W(x)=B_{m}(x) /\left[A_{m}(x)-B_{m}(x)\right]
$$

$$
\begin{aligned}
\left(a_{0}-b_{0}\right) w_{i}+\left(a_{1}-b_{1}\right) w_{i+1}+\ldots+a_{m} w_{i+m} & =0, \\
i>0, a_{m} & \neq 0,
\end{aligned}
$$

as required. The argument is clearly applicable to prove the converse part of the theorem.

Although the next example is very simple it can easily be shown that in it the elements of $\{w\}$ do not satisfy any linear recurrence with constant coefficients.

EXAMPLE 5. $\quad \mathrm{w}_{\mathrm{n}}=(-1)^{\mathrm{n}+1} / \mathrm{n}$ ! In this case $w(x)=1-e^{-x}$ and $w(x)=e^{x}$ so that $W_{n}=1 / n$ !

We conclude with several examples of a more complicated nature, leaving a comment about proof to the end.

EXAMPLE 6. For every $a \neq 0, a \neq 1, w_{n}=\frac{a-1}{a} \cdot \frac{1}{n a-1}\left(\begin{array}{c}n a \\ n\end{array}\right)$ 
if and only if $W_{n}=\frac{1}{n}\left(\begin{array}{c}n a \\ n-1\end{array}\right)$. This may be obtained from theorem 1 by letting $w(x)$ be the function determined by

$$
x=w(x)(1-w(x))^{a-1}
$$

EXAMPLE 7. For every $a \neq 0, a \neq 1, w_{n}=\frac{a-1}{n a-1}\left(\begin{array}{c}n a \\ n\end{array}\right)$ if and only if $w_{n}=\left(\begin{array}{c}n a \\ n\end{array}\right)$. We obtain this result by considering the function $w(x)$ determined by

$$
x=\frac{w(x)}{a}\left(1-\frac{w(x)}{a}\right)^{a-1}
$$

Note that in the last two examples the sequences $\{w\}$ and $\{W\}$ consist of integers if a is an integer. The fact that if either of the sequences consist of integers then both must be of this form follows directly from (1) and theorem 2 .

Our final example makes use of the inversion of the function $x=w(x) e^{-w(x)}$.

EXAMPLE 8. $w_{n}=\frac{n^{n-1}}{n !}$ if and only if $w_{n}=\frac{n^{n}}{n !}$. This is. obtained by considering the function $w(x)$ determined by

$$
x=w(x) e^{-w(x)}
$$

The results of the last three examples involve an application of Lagrange's coefficient formula for Taylor series expansions, namely: if $y=w(x), w_{0}=0, w_{1} \neq 0$ then

where

$$
f(y)=\Sigma_{i=-\infty}^{\infty} c_{i} x^{i}
$$

$$
\frac{f^{\prime}(y)}{x^{n}}=\ldots+\frac{n c_{n}}{y}+\ldots
$$

as a Laurent series in $y$. Thus

if

$$
\begin{aligned}
\frac{1}{x^{n}} & =\ldots+\frac{n c_{n}}{y}+\ldots \\
y & =\Sigma_{i=1}^{\infty} w_{i} x^{i}=w(x) .
\end{aligned}
$$

University of Alberta 